\title{
RECENT ADVANCES IN SYNTHETIC CALIBRATIONS OF MULTI JUNCTION SOLAR CELLS AND THEIR CORRESPONDING COMPONENT CELLS
}

\author{
Florian Witt $^{(1)}$, Ingo Kröger ${ }^{(1)}$, Stefan Winter ${ }^{(1)}$ \\ (1) Physikalisch-Technische Bundesanstalt Braunschweig, Bundesallee 100, 38114 Braunschweig, Germany \\ Florian.Witt@ptb.de
}

\begin{abstract}
Precise calibrations of primary reference standards for Multi Junction Solar Cells are crucial for the exact characterization of photovoltaic devices for space applications. To complement the established but rarely performed atmospheric calibration campaigns via balloon flights a reliable laboratory based, synthetic, calibration method is desirable. Differential spectral responsivity measurements are an established calibration method for solar cells in terrestrial application. Here it is shown that such synthetic calibrations can also be performed with lowest measurement uncertainties on Component Cells but also on whole Multi Junction Solar cell devices.
\end{abstract}

\section{INTRODUCTION}

The quality control in the field of Multi Junction Solar Cells (MJSC) for space applications relies until now on primary reference standards which are directly measured close to AM0 like conditions at high altitudes [1]. A disadvantage of such calibration campaigns is the long time between the flights, for Europe no mission was performed up to now since 2005. Another drawback are the high costs of such a single flight as well as the risks of failure and loss of samples. These points can be addressed by the application of a laboratory based, synthetic, calibration method. For solar cells used in terrestrial applications differential spectral responsivity (DSR) calibrations have been established as method with lowest measurement uncertainties for the short circuit current under terrestrial $\left(1000 \mathrm{~W} / \mathrm{m}^{2}\right.$ irradiance with an AM1.5 spectrum at $25 \mathrm{C}^{\circ}$ ) standard test conditions obtainable [2]. This method has also been applied to determine the short circuit current under space test condtions $\left(I_{\mathrm{STC}}\right)$ of Component Cells (a reference solar cell where only a single junction of a MJSC device is electrically active) but with higher measurement uncertainties as for silicon reference solar cells [3]. The space-like test conditions were defined as follows: $\sim 1360 \mathrm{~W} / \mathrm{m}^{2}$ irradiance with an AM0 spectrum (according to Wehrli [4]) at $25 \mathrm{C}^{\circ}$. Within the European Metrology Research Program (EMRP) project SolCell methods and devices to improve DSR calibrations of Component as well as MJSC solar cells are developed. In this work recently obtained results are presented.

\section{THE DIFFERENTIAL SPECTRAL RESPONSIVITY CALIBRATION METHOD}

The short circuit current of any solar cell is determined by the cell's absolute spectral responsivity $(s(\lambda)$ with $\lambda$ as wavelength) and the spectral irradiance $\left(E_{\mathrm{AMx}}(\lambda)\right.$ with $\mathrm{x}=0$ or $\mathrm{x}=1.5$ respectively) at which the cell is measured (equation 1). Therefore, to determine precisely the $I_{\mathrm{STC}}$ value of a solar cell under a given reference spectrum a precise determination of its spectral responsivity is necessary.

$$
I_{\mathrm{STC}}=\int s(\lambda) \cdot E_{\mathrm{AMx}}(\lambda) \mathrm{d} \lambda .
$$

The absolute spectral responsivity is determined in the following way. A monochromatic modulated probe beam generates a small $\mathrm{AC}$ current in the device under test (DUT) which is kept close to short circuit conditions. The wavelength of the beam is varied and the current answer in dependence of the wavelength is determined via a trans-impedance/ lock-in amplifier combination. In parallel, the DUT is illuminated by a constant DC bias light field. To consider the inhomogeneity of the DUT as well as the influence of the sample's housings, all measurements are performed with both light fields over illuminating the sample. Due to the modulation of the monochromatic irradiance, the measured quantity is the differential spectral $\widetilde{s}\left(\lambda, I_{\text {bias }}\right)$. Therefore it is necessary to perform the measurements at different irradiance levels below and above the targeted test conditions (either $1000 \mathrm{~W} / \mathrm{m}^{2}$ for AM 1.5 like conditions or the integrated irradiance value $\left(\sim 1360 \mathrm{~W} / \mathrm{m}^{2}\right)$ of the applied AM0 spectrum). Here $I_{\text {bias }}$ corresponds to the DC current the solar cell is generating at the given DC bias light field [5].

The absolute irradiance of the monochromatic light field is determined by a primary calibrated irradiance standard traced to a cryogenic radiometer. This reference is measured subsequently to the DUT. Due to the monitor based measurement design these independent measurements can be directly compared. To determine the absolute spectral responsivity from the differential method at first a differential (non-spectral) AM0 weighted responsivity $\widetilde{S}\left(I_{\text {bias }}\right)$ is needed. It is 
obtained by equation 2 .

$$
\widetilde{s}\left(I_{\text {bias }}\right)=\frac{\int_{0}^{\infty} \widetilde{s}\left(\lambda, I_{\text {bias }}\right) \cdot E_{\lambda, \mathrm{AM} 0}(\lambda) \mathrm{d} \lambda}{\int_{0}^{\infty} E_{\lambda, \mathrm{AM} 0}(\lambda) \mathrm{d} \lambda}
$$

If the investigated solar cells would be linear, the short circuit current under given test conditions could be simply calculated from a single $\widetilde{s}\left(I_{\text {bias }}\right)$. Due to the in reality occurring non-linear behavior, it becomes necessary to solve equation 3 to obtain the $I_{\mathrm{STC}}$ value of the DUT.

$$
E=\int_{0}^{I_{\mathrm{STC}}} \frac{1}{\widetilde{s}\left(I_{\text {bias }}\right)} \mathrm{d} I_{\text {bias }}
$$

From this equation it becomes apparent that the $I_{\mathrm{STC}}$ is defined implicitly as integration limit. Equation 3 is solved for $I_{\mathrm{STC}}$ therefore iteratively by increasing the $I_{\mathrm{STC}}$ value until $E$ equals the absolute irradiance of the given reference spectrum.

$$
s\left(\lambda, I_{\mathrm{STC}}\right)=\frac{I_{\mathrm{STC}}}{\int_{0}^{I_{\mathrm{STC}}} \frac{1}{\widetilde{s}\left(\lambda, I_{\text {bias }}\right)} \mathrm{d} I_{\text {bias }}}
$$

When the $I_{\mathrm{STC}}$ value is obtained, also the absolute spectral responsivity at the given test conditions can be subsequently calculated from equation 4 .

\section{THE LASER DSR-FACILITY}

For the measurements presented in this work, a tunable laser source was used as monochromatic radiation source (figure 1). In contrast to standard DSR setups basing on classical light sources combined with monochromators, this setup possesses a higher spectral irradiance. This is used on the one hand to improve the signal to noise ratio of the modulated signal and on the other hand to increase the homogeneity and size of the monochromatic light field. As shown in [6] inhomogeneity as well as wavelength deviations of the monochromatic light field dominate the overall measurement uncertainty of $s(\lambda)$ and $I_{\mathrm{STC}}$. With the use of multiple diffusors and a wavelength dependent positioning of the imaging optics the inhomogeneity is kept below $1 \%$ for all wavelengths in the area of the sample. For further reducing the influence of the light's field inhomogeneity the reference scans the light field for every wavelength in the whole area of the DUT. To address the issue of wavelength deviations a calibrated array-spectroradiometer is used for live monitoring the monochromatic signal.

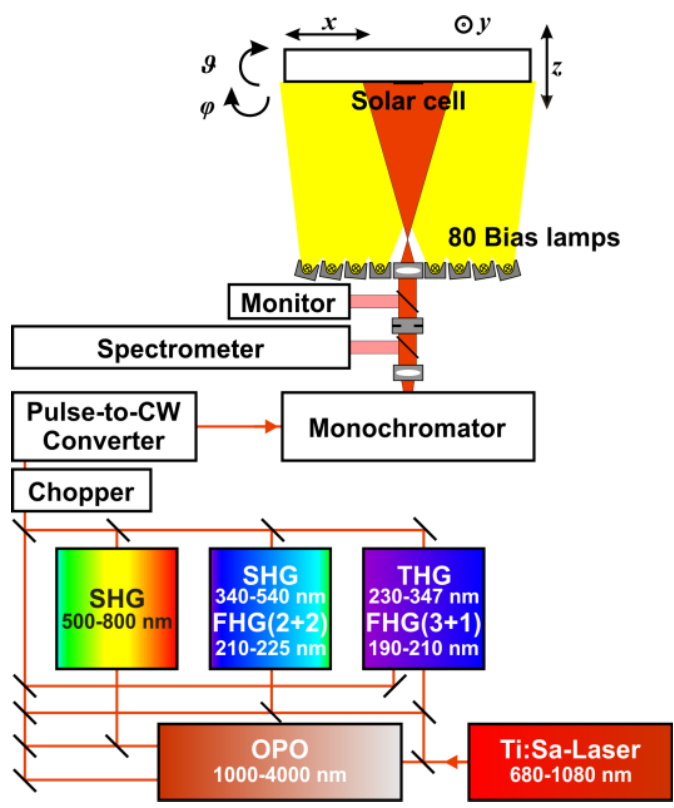

Figure 1: Optical setup used for laser-based DSR measurements. A tunable Ti:Sa-Laser in combination with multiple frequency multipliers as well as an optic parametric oscillator allow an accessible spectral range from $190 \mathrm{~nm}$ to $4000 \mathrm{~nm}$.

The temperature control of the samples is realized with Peltier elements which can directly cool or heat the DUT under illumination. The stability in temperature is found commonly in the range of millikelvins. The temperature of the DUT can be chosen freely and is commonly set to $25^{\circ} \mathrm{C}$ according to terrestrial standard test conditions. The temperature coefficient of $I_{\mathrm{STC}}$ is determined additionally by DSR measurements at three different temperatures.

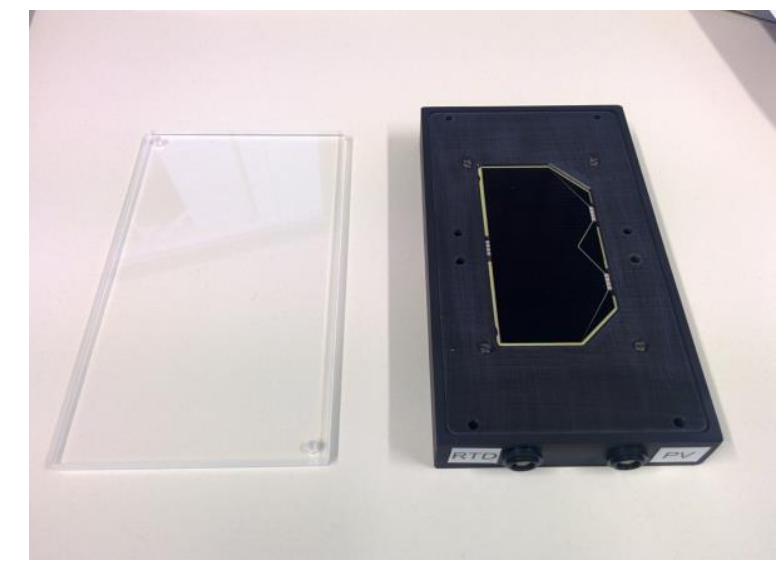

Figure 2: A reference component solar cell in its WPVS compatible customized housing. Connectors for solar cell and temperature sensor positioned at the bottom side. Cover shield for transportation and storage on the left. 


\section{SYNTHETIC CALIBRATION OF COMPONENT SOLAR CELLS}

The described procedure was applied to standard triple junction component solar cells composed of a InGaP top cell, an InGaAs middle Cell and a Germanium bottom cell. In each of the three investigated devices only one of the three sub junctions were electrically active. The cells were built into WPVS compatible housings (see figure 2). Close to the solar cell a PT 100 temperature sensor was attached. In figure 3 differential spectral responsivity measurement results of each DUT is shown for selected bias light levels. A first observation here is that the devices $\widetilde{s}\left(\lambda, I_{\text {bias }}\right)$ vary only little with the DC irradiance.
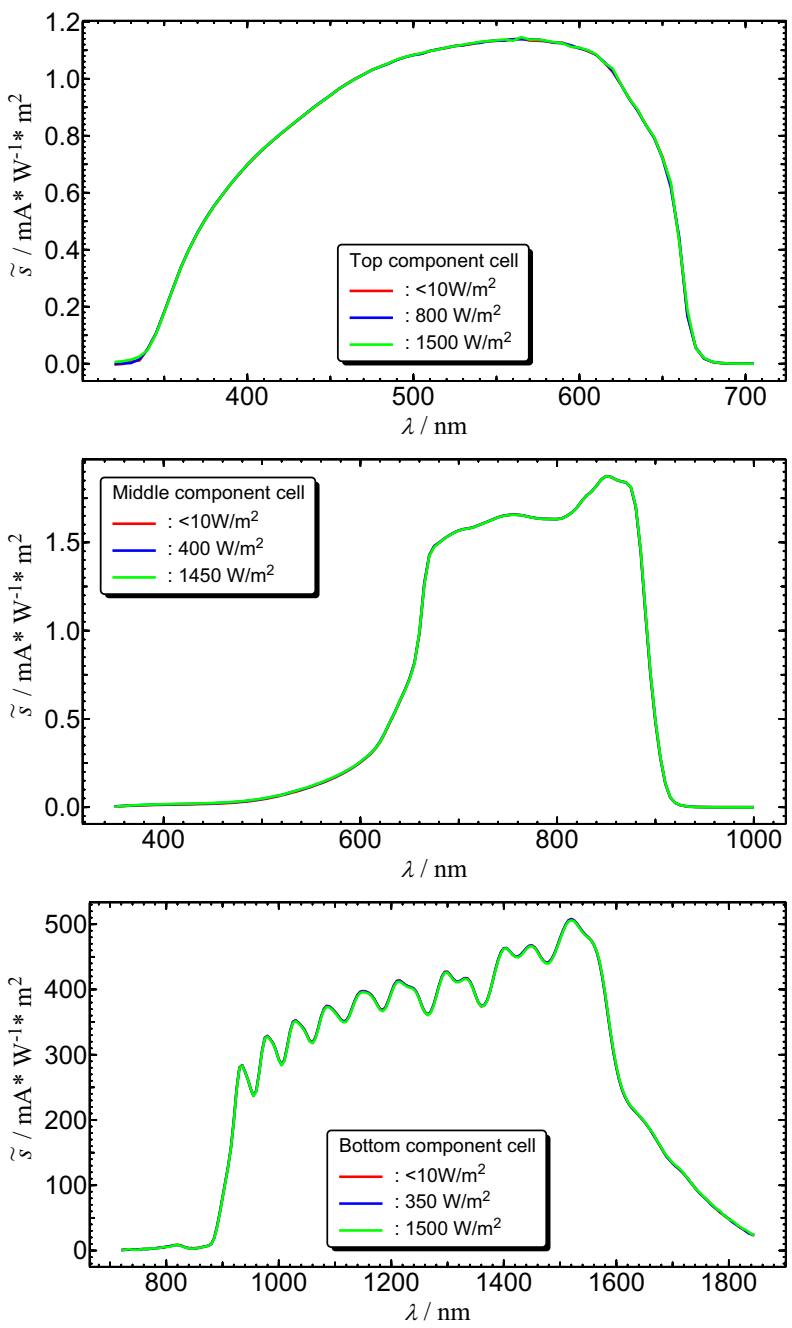

Figure 3: The differential spectral responsivity of each investigated component cell is plotted at three different DC light bias irradiance levels.

This is further supported by the plot of the AM0 weighted differential responsivity (calculated from equation 2) of all measured bias light levels in figure 4 .
According to equation 3 and 4 the $I_{\mathrm{STC}}$ and the absolute spectral responsivity $s(\lambda)$ is calculated (latter shown in figure 5). With a Monte Carlo simulation (for details see [6]) the measurement uncertainties of all calibration results are determined. For the top and middle component cells a relative measurement uncertainty (MU) of the $I_{\mathrm{STC}}$ of $0.5 \%(k=2)$ was achieved. In the case of the bottom junction component solar cell $1 \%$ relative measurement uncertainty $(k=2)$ for the $I_{\mathrm{STC}}$ was achieved. Here the calibration uncertainty of the used germanium irradiance standard was found as one of the main limiting factors.
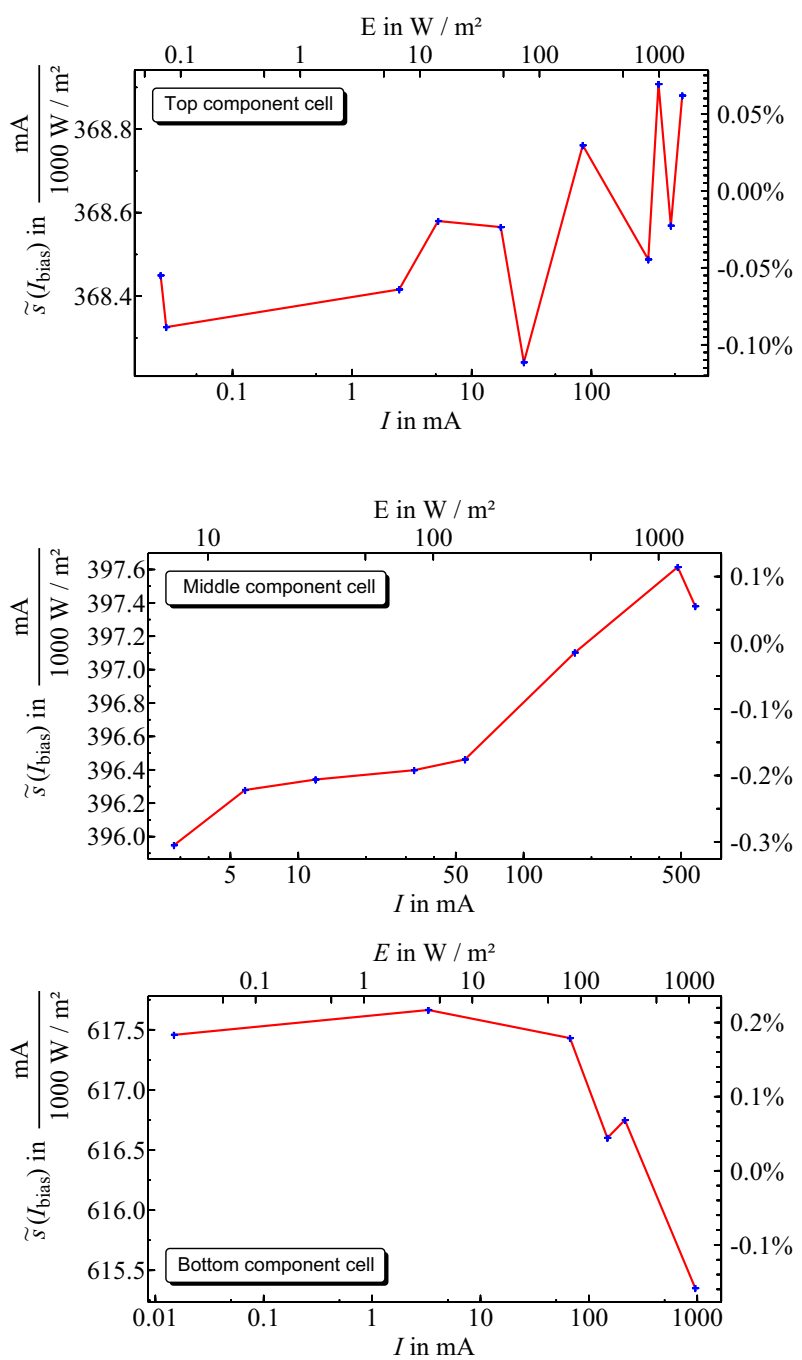

Figure 4:AM0 weighted DSR $\widetilde{s}\left(I_{\text {bias }}\right)$ calculated from the AMO reference spectrum and the $\widetilde{s}\left(\lambda, I_{\text {bias }}\right)$ measurements for all investigated component cells. Here I corresponds to the measured DC current of the DUT which is later converted to the corresponding irradiance level E. The integral non-linearity of all samples is below $0.5 \%$.

This important result states that it is now possible to achieve a MU on the same order of magnitude as 
obtainable by balloon flight calibrations.

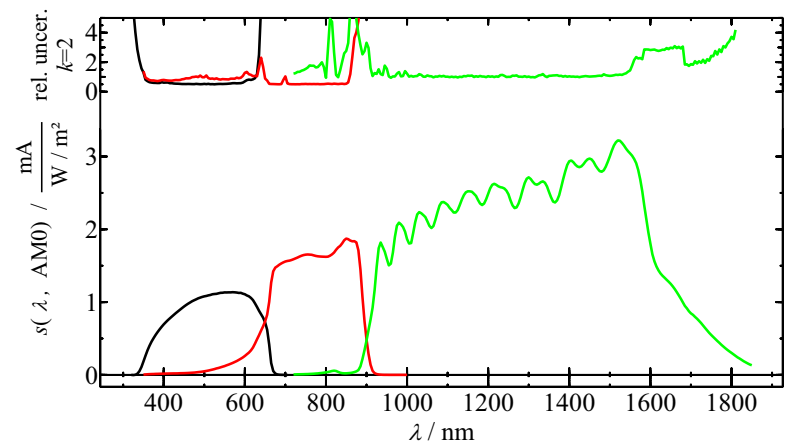

Figure 5: Spectral Responsivity at AMO test conditions of Top Comp. Cell (black), Mid Comp. Cell (red) and Bottom Comp. Cell (green) and the associated relative measurement uncertainty.

\section{SYNTHETIC CALIBRATION OF MULTI- JUNCTION SOLAR CELLS}

The application of the DSR method on MJSC is a nontrivial experiment. Due to the serial connection of the sub junctions it is impossible to generate an AC current by exciting a single sub junction monochromatically. To solve this issue and to determine the relative DSR from a MJSC device it is established to apply polychromatic bias lights which are chosen so that for each sub junction of the device an own DC excitation source is available [7]. These sources are adjusted so that currents in the sub junctions not under test are generated in excess compared to the junction under test. In this way a modulated current generated in the targeted sub junction can also be measured over the whole device. Similar to the investigations on the component cells different $\mathrm{dc}$ irradiation levels were defined, so that the junction under test was operated below and above $1366 \mathrm{~W} / \mathrm{m}^{2}$. In figure 6 the application of such polychromatic light sources is shown. Here high power LEDs $(470 \mathrm{~nm}$ LEDs for the excitation of the top junction and $780 \mathrm{~nm}$ LEDs for the excitation of the middle junction are used. The bottom junction is excited by halogen lamps). Additionally, it is necessary to apply a constant DC offset voltage since by the polychromatic illumination the junction under test is shifted to negative operation voltages.

Results from the measurements regarding the top and middle sub junction of the DUT are shown in figure 7. Here the voltage dependence of the DSR at a single polychromatic bias light combination is shown.

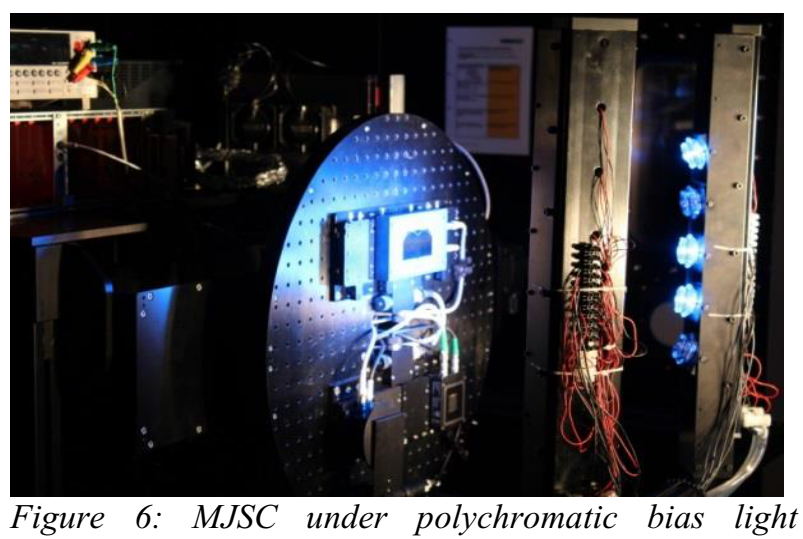
illumination during laser-DSR calibration.

As can be seen the voltage has only a slight influence on the DSR. To take the slight voltage dependence into account the $\widetilde{s}\left(\lambda, I_{\text {bias }}\right)$ measurements were performed at three different voltage levels and subsequently averaged before $\widetilde{s}\left(I_{\text {bias }}\right)$ was calculated. In figure 8 $\widetilde{s}\left(\lambda, I_{\text {bias }}\right)$ as well as the calculated $s(\lambda)$ are shown.
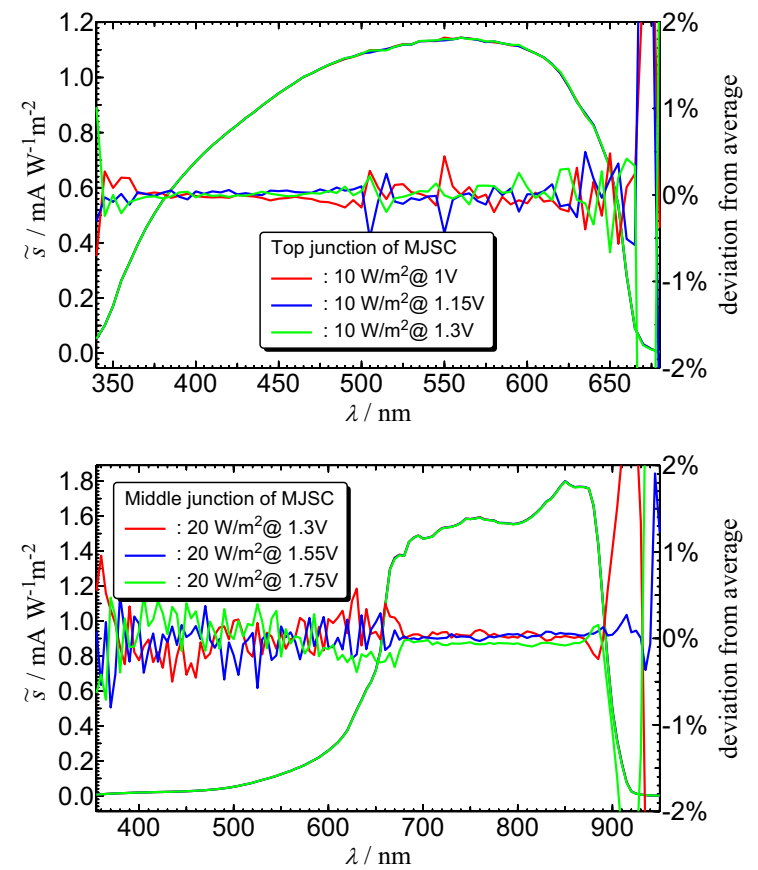

Figure 7: Voltage dependence of Top and Middle junction DSR of MJSC at fixed bias light level. The annotated irradiance level corresponds to the irradiance from the current limiting light source (For the top junction the $470 \mathrm{~nm}$ LEDs, for the middle junction the $780 \mathrm{~nm}$ LEDs).

As observed with the component cells before, the sub junctions show a linear behaviour. The $I_{\mathrm{STC}}$ value of the top sub junction was obtained with a relative uncertainty of about $1 \%$. For the middle junction similar results are obtained. 

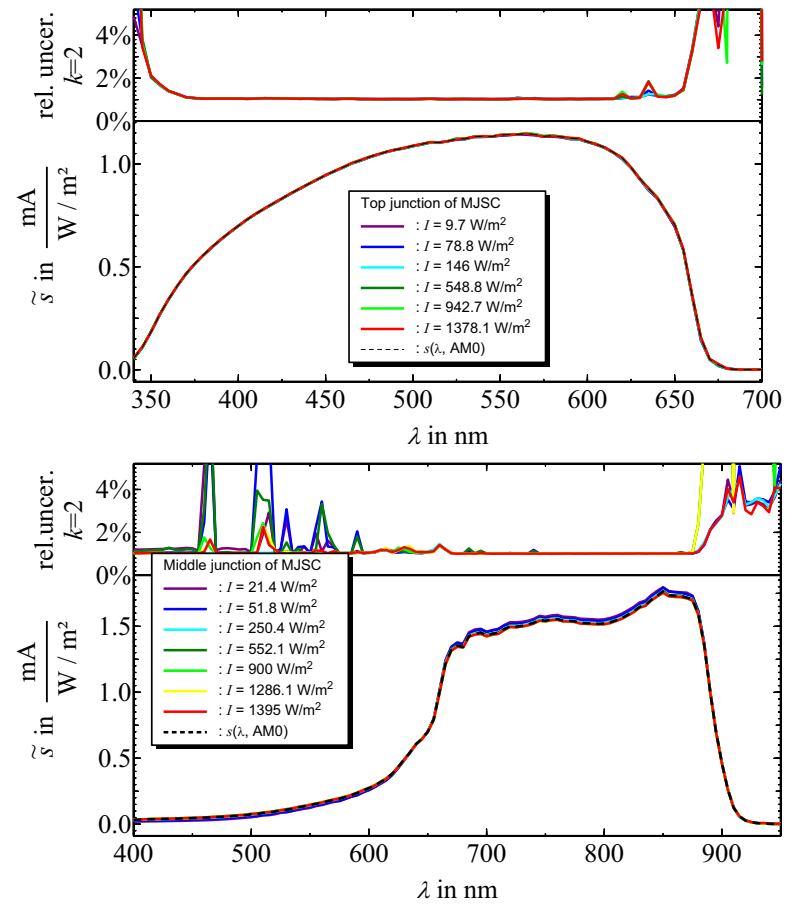

Figure 8: $\widetilde{s}\left(\lambda, I_{\text {bias }}\right)$ of top and middle junction of MJSC as well $s(\lambda)$. Additionally, the calculated relative $M U$ is shown.

During measurements of the bottom junction issues related of a parasitic parallel resistance was observed as reported in literature. E.g. an artefact of the middle sub junction of the device was found in the bottom sub junction signal and the DSR of the bottom junction was suppressed (upper graph in figure 9). As expected it was not possible to suppress the artefact by the variation of the dc bias voltage.

To correct the measured data a method published by Siefer et al in [8] was applied to the obtained DSR results. In the lower graph of figure 9 the corrected data are presented. It is notable that almost no non-linearity at different bias light levels is observed anymore, as expected from experiments on bottom component cells. Experimentally due to insufficient irradiance from the polychromatic bias light it was only possible to measure the DSR of the bottom sub junction at an estimated absolute irradiance of about $500 \mathrm{~W} / \mathrm{m}^{2}$. A preliminary evaluation results in an $I_{\mathrm{STC}}$ value comparable to the currents generated in bottom junction component cells. Here further work is necessary to verify these findings and to achieve DC bias light irradiance levels sufficient to generate currents above the expected $I_{\mathrm{STC}}$ in the bottom junction of the MJSC device.
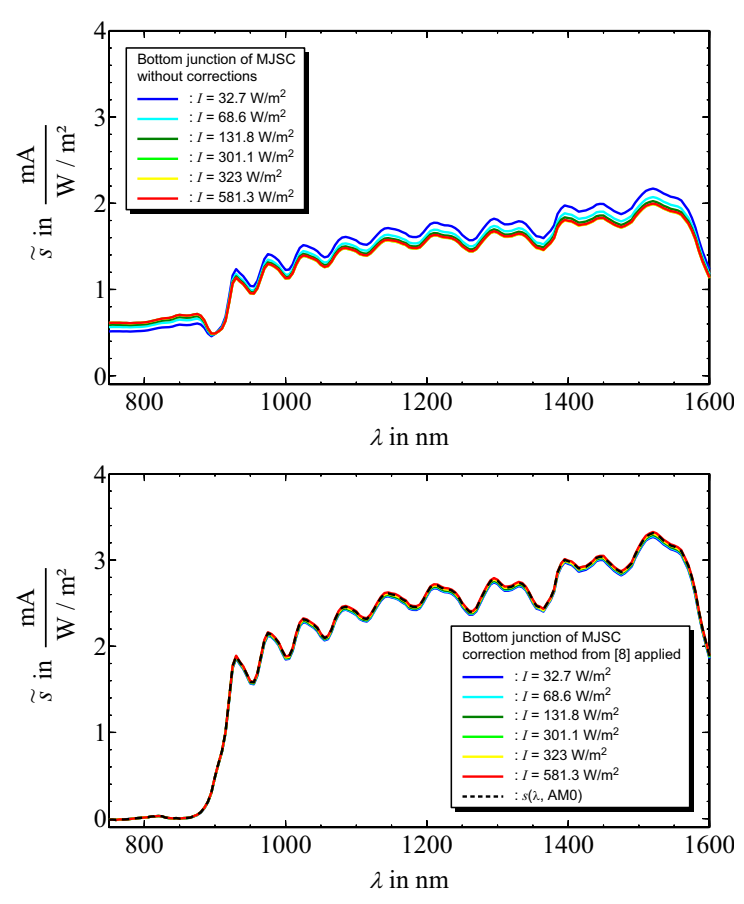

Figure 9 Top: DSR results from the polychromatic measurement of the bottom sub junction of the MJSC. An artefact of the middle junction and a large nonlinearity in irradiance of the sub junction is observed. Bottom: After applying the correction procedure from [8] all measurements, show the expected linear behaviour.

\section{CONCLUSION}

In this work we presented the results from a calibration campaign which states that synthetic calibration of MJSC and their corresponding component cells can be performed and achieve measurement uncertainties comparable to balloon flight calibrations. With these tools in future, a reliable and less expensive method for Si-traceble primary calibrations of component cell references in the field of space photovoltaics is available. To give further prove of the suitability of this method for the quality control of component cells used as primary working standards a direct comparison to balloon flights need to be addressed in future.

\section{ACKNOWLEDGEMENTS}

We thank AZUR SPACE for the supply with samples. The research work leading to this article was carried out within the EMRP ENG51 project SolCell. The EMRP is jointly funded by the EMRP participating countries within EURAMET and the European Union. 


\section{REFERENCES}

1. Pichetto, V. (2002). CASOLBA Calibration of Solar Cells-Measurement and Calibration Procedures. InProc. 29'" IEEE Photovoltaics Specialists Conference., New Orleans (p. 1010)

2. Winter, S., Metzdorf, J., Emery, K., Fabero, F., Hishikawa, Y., Hund, B., ... \& Warta, W. (2005). The results of the second world photovoltaic scale recalibration. In Photovoltaic Specialists Conference, 2005. Conference Record of the Thirty-first IEEE (pp. 1011-1014). IEEE.

3. Baur, C., Siefer, G., Kern, R., \& Winter, S. (2011). Primary Solar Cell Standards-Comparison Of Extraterrestrial And Synthetic Calibration. In ESA Special Publication (Vol. 690, p. 12).

4. Wehrli, C. (1985) "Extraterrestrial Solar Spectrum", Publication no. 615, PhysikalischMeteorologisches Observatorium + World Radiation Center (PMO/WRC) Davos Dorf, Switzerland.

5. S. Winter, T. Wittchen, J. Metzdorf (2000)

"Primary Reference Cell Calibration at the PTB

Based on an Improved DSR Facility"; in "Proc.

16th European Photovoltaic Solar Energy

Conf.",(p.4).

6. Winter, S., Fey, T., Kröger, I., Friedrich, D., Ladner, K., Ortel, B., ... \& Witt, F. (2014). Design, realization and uncertainty analysis of a laser-based primary calibration facility for solar cells at PTB. Measurement, 51, (pp. 457-463).

7. Meusel, M., Baur, C., Létay, G., Bett, A. W., Warta, W., \& Fernandez, E. (2003). Spectral response measurements of monolithic GaInP/Ga (In) As/Ge triple-junction solar cells: Measurement artifacts and their explanation. Progress in Photovoltaics: Research and Applications, 11(8), (pp.499-514).

8. Siefer, G., Baur, C., \& Bett, A. W. (2010). External quantum efficiency measurements of germanium bottom subcells: Measurement artifacts and correction procedures. In Photovoltaic Specialists Conference (PVSC), 2010 35th IEEE (pp. 000704000707). IEEE. 